\title{
Dentistry concerns for patients taking anticoagulants and antiplatelet drugs
}

\section{Paul Sambrook}

Chair, Dental Therapeutics

Committee, Australian

Dental Association, Sydney

Aust Prescr 2018:41:116

https://doi.org/10.18773/

austprescr.2018.040
Dentists must be aware of all patients who are being treated with oral anticoagulants or antiplatelet drugs. Some patients may be taking anticoagulants in combination with antiplatelet drugs. There should be an understanding as to why these drugs have been prescribed.

The risk of coronary events has to be weighed against the risk of bleeding. ${ }^{1,2,3}$ The regimen will have been individualised with a careful assessment of each patient's risk of thrombosis and bleeding to find the optimal balance between harm and benefit. In most instances of dental treatment, the regimen should not be interfered with as this could place the patient at greater risk.

For simple surgical procedures such as the extraction of a single tooth or scaling and cleaning, alteration of the regimen is not required. After an extraction local measures such as haemostatic material supplemented by a suture is all that is needed.

If more extensive surgery is contemplated then a risk assessment in conjunction with the patient's

\section{REFERENCES}

1. Breik O, Cheng A, Sambrook P, Goss A. Protocol in managing oral surgical patients taking dabigatran. Aust Dent J 2014;59:296-301. https://doi.org/10.1111/adj.12199

2. Oral and dental. In: eTG complete [Internet]. Melbourne: Therapeutic Guidelines Limited; 2018. www.tga.org.au [cited 2018 Jul 1] medical practitioner must be undertaken. If it is felt that the risk of stopping the drugs is great, surgery must be modified. Is the risk of not having the surgery greater than the risk of cardiovascular events? Can the surgery be spread over a number of appointments? If surgery cannot be postponed or split into shorter sessions, inpatient management would generally be required.

After surgery the patient must be given explicit, written instructions. The patient must be watchful for unusual or prolonged bleeding and know where and how to receive advice and help.

The message here is that stopping the drugs is often more risky than the issue of bleeding. In most instances minor surgery combined with local haemostatic measures is required. If alteration of the antithrombotic regimen is required, this must be done in a monitored and controlled environment.

Conflict of interest: none declared
3. Tran H, Joseph J, Young L, McRae S, Curnow J, Nandurkar H, et al. New oral anticoagulants: a practical guide on prescription, laboratory testing and peri-procedural/ bleeding management. Intern Med J 2014;44:525-36 https://doi.org/10.1111/imj.12448 